Aus dem Medizinhistorischen Institut der Universität Basel

Vorsteher: Prof. Dr. med. H. Buess

\title{
Theodor Meyer-Merian (1818-1867), Arzt, Spitalmeister und Volksdichter
}

\author{
zu seinem 100.Todestag am 5. Dezember $1967^{1}$
}

\author{
Von C. Hodel, New Haven
}

\begin{abstract}
Einleitung
Ende des letzten Jahrhunderts, ja noch vor fünfzig Jahren, wäre es nicht nötig gewesen, Theodor Meyer-Merian vorzustellen; galt er doch als einer der bekanntesten und vor allem in einfacheren Kreisen beliebtesten schweizerischen Volksschriftsteller. Wer das Vergnügen hat, auf die interessante und reiche Persönlichkeit Meyers zu stoßen, kann seinen Schriften eine Fülle von lehrreichen und amüsanten Informationen und Berichten aus dem Leben des Bürgers einer Kleinstadt im letzten Jahrhundert entnehmen. Darüber hinaus hat uns Meyer-Merian einige wertvolle und zeitlose Gedanken über das Arzttum hinterlassen, die auch heute noch ihre Gültigkeit besitzen. Es scheint deshalb gerechtfertigt, anläßlich seines 100.Todestages Meyer-Merian der Vergessenheit zu entreißen und einige seiner Worte aus den zahlreichen Schriften wieder aufleben zu lassen.
\end{abstract}

\section{Sein Leben}

Th. Meyer-Merian wurde am 14.Januar 1818 im Haus «Zum goldenen Falken » an der unteren Freiestraße geboren (heute Haus Nr.9). Ganz in der Nähe seines Geburtshauses stand damals der Dalilabrunnen, welcher heute am Barfüßerplatz steht. Die damalige Freiestraße hatte einen sehr kleinbürgerlichen, gemütlichen Charakter, welcher erst im späteren Leben Meyers durch tiefgreifende Sanierungen zerstört wurde. Sein Vater Daniel Meyer, von Beruf Kaufmann, gehörte dem längsteingesessenen Geschlecht der Meyer «zum Pfeil» ${ }^{2}$ an.

Seine Mutter Katharina war eine geborene Hofmann.

1 Für den Druck durchgesehen und ergänzt von Dr. M.-L. Portmann.

2 Altes Basler Achtburger- und Ratsgeschlecht, das wohl schon vor 1356 das Bürgerrecht erwarb. 
Theodor wird als kecker, frischer und intelligenter Junge beschrieben, der schon früh eine gewisse Selbständigkeit erwarb. Die Kinder tummelten sich auf den Straßen und in den Stadtgräben. Wie alle Jungen machte Theodor begeistert im Kadettenwesen und im Knabenturnverein mit. Nachmittage bei der Großmutter im Gartenhaus vor dem Spalentor gehörten zu den regelmäßigen, rituellen Ausflügen am Donnerstag, welche ihren Niederschlag in späteren Schriften gefunden haben.

Theodor besuchte das Pädagogium « auf Burg». Er war ein eifriger Turner und wurde später Mitglied der «Zofingia». Während der Schulzeit stand er stark unter dem Einfluß des Germanisten Wilhelm WackernageL (1806-1869), dessen Name mit seiner deutschen Literaturgeschichte verbunden ist.

Wackernagel beeinflußte einen Freundeskreis um M. so stark, daß ein von den Schülern gegründetes Literaturkränzchen feste Formen annahm. Die Freunde, unter ihnen J. J. Oeri (später Pfarrer in Lausen), Alois BiedermanN (später freisinniger Theologe), J. J.Bosshardt, J. und Th. Burckhardt, Friedrich Oser (später Pfarrer in Benken) und Johann RiggenBACH (später Theologieprofessor), trafen sich allwöchentlich zum «Vortrag» eigener Werke oder ausgewählter Vorbilder. Auch unterhielten sie eine eigene Zeitung: die Euterpe. Die starke Bindung, welche von einer solchen Jugendfreundschaft und Betätigung ausging, hat bei den meisten Beteiligten das ganze Leben angedauert. Aus der Feder seiner Freunde haben wir auch die getreuesten Lebensbeschreibungen von Theodor. Meyer scheint harmlos-witzig und manchmal ironisch gewesen zu sein; doch ist leider von ihm aus jener Zeit kaum etwas erhalten. Er entwickelte damals eine große Liebe zur Theologie und eine starke Bindung zu J.P. HeBEL, zu dessen Ehren er eine, allerdings nicht gehaltene Maturrede verfaßt hat. Nach Schulabschluß entschloß er sich als einziger seiner Freunde für die Medizin und bezog im Jahre 1837 die Universität. Die ersten fünf Semester verbrachte er an der Basler medizinischen Fakultät mit ihren vier Lehrstühlen: für Anatomie, Physiologie und Pathologie, Chirurgie und Botanik.

Nach den Vorlesungen in kleinem Kreise folgte meist ein erholsamer Spaziergang über den Münsterplatz. Seine Professoren hat Meyer in trefflichen Karikaturen festgehalten, welche zum Teil noch in Privatbesitz vorhanden sind.Obwohl er der einzige Mediziner war, fühlte sich Theodor seinem Freundeskreis, welcher übrigens gesamthaft der Studentenverbindung «Zofingia » beigetreten war, nach wie vor verbunden. Die Tiefe solcher Biedermeierfreundschaften läßt sich daran ermessen, daß sich Theodor 
während der folgenden zwei Semester in Freiburg im Breisgau, wie er selber schreibt, sehr einsam und unglücklich fühlte.

Während seiner Studienzeit entwickelte er eine realistische Denkweise und wurde Gegner alles Spekulativen. Dies ist vor allem darum bemerkenswert, weil seine anschließende Bildungsreise durch Deutschland - sie führte ihn bis an die berühmte Charité in Berlin zum Internisten J.L.SchoenleIN (1793-1864) und zum Chirurgen J.F.DiefFenBAGH (1792-1847) - ganz die Romantik spiegelt: Die Wanderlust und das Reisen mit der Postkutsche, das einfache, aber gemütvolle Leben und tiefe, stark von der Natur geprägte Empfindungen, welche sich aber offenbar nicht in die unbestimmte Spekulation verlieren mußten.

Als Meyer nach Basel zurückkehrte, zog er in die neue Wohnung seiner Eltern am Sankt-Johann-Tor. Er schloß sein Studium im Jahre 1841 mit einer Dissertation über das Carcinom der Leber ab. Anschließend wurde er Assistenzarzt beim Internisten C.G.Jung (1795-1864), welcher die medizinische Fakultät über ihre kritische Zeit gerettet hatte. In dieser Zeit entstanden seine ersten Gedichte. Im Jahre 1846 eröffnete er eine eigene Praxis und habilitierte sich als Privatdozent. In das gleiche Jahr fällt die Vermählung mit Eleonore Merian «vom rothen Hause». Fortan führte Theodor den Doppelnamen Meyer-Merian. Das Paar bezog eine Wohnung am Nadelberg, gegenüber der damaligen Almosenschaffnerei. Der kurz nach seiner Hochzeit ausbrechende Sonderbundskrieg sah Meyer als Arzt des städtischen Infanteriekorps. Die «Briefe eines Arztes», welche um 1850 geschrieben wurden, lassen erkennen, daß Meyer wegen seiner derben Geradheit in der Praxis nicht restlos glücklich war, weil er sich den Launen seiner Kranken einerseits nicht anpassen konnte und andererseits oft die Hilflosigkeit des Arztes erkennen mußte. Zudem scheint die schriftstellerische Begabung immer mehr zum Durchbruch gekommen zu sein; doch ließ ihm die Arztpraxis dazu zu wenig Zeit. So bewarb er sich im Jahre 1851 um die freigewordene Stelle eines Spitalmeisters. Aus 14 Bewerbungen Kaufleute, Postbeamte, Pfarrer und Kommissäre hatten sich für die Stelle interessiert - ging er als Sieger hervor.

Das Spital, das neben dem Barfüßerkloster in der zweiten Hälfte des 13. Jahrhunderts als ein Werk der Bürgerschaft gegründet wurde, war in der Tradition der Stadt Basel fest verwurzelt. Die Oberaufsicht führte das Pflegamt, das aus Mitgliedern der Bürgerschaft bestand und die Spitalverwaltung zu prüfen hatte. Die geschäftliche und administrative Leitung übte der Spitalmeister aus. Diese Regelung der Spitalverwaltung war schon 
im 13. Jahrhundert getroffen worden. Vor allem waren es Arme und Bedürftige, die im Spital Aufnahme fanden. Diesen Charakter behielt das Spital bis ins 19. Jahrhundert hinein bei. Unter tatkräftiger finanzieller Mithilfe der Bürgerschaft wurde das Spital 1838-1842 an die Hebelstraße verlegt; und zwar wurde der Markgräflerhof als Pfrundhaus verwendet, und ein ihm angeschlossener Neubau umfaßte das Kranken- und Irrenhaus. Hier war nun also die neue Wirkungsstätte von Meyer.

Seine neue Stellung verlangte Organisationstalent, Takt im Umgang mit Menschen und einen gut entwickelten Sinn für Gemeinnützigkeit und Volkswohlfahrt. Ein vollbepacktes Arbeitsbündel wartete auf ihn: Die Reorganisation des Spitalwesens mit Plänen zur baulichen Vergrößerung (!), die Eindämmung der Typhus- und Choleraepidemien in den fünfziger Jahren und andere sozialhygienische Fragen. Daneben schrieb Meyer eine ganze Reihe von Dramen, Romanen und Gedichten. Sein schriftstellerisches Talent entfaltete sich aber erst so richtig voll in seinen historischen Aufsätzen und seinen Kalendergeschichten. Von 1853 bis 1856 stand er als Herausgeber dem Schweizerischen Hausboten vor und 1860 bis 1865 dem Kalender des Basler Hinkenden Boten. Der Einfluß J.P.Hebels wird hier unverkennbar.

Das Elend, das er als mitfühlender Arzt und Spitalmeister täglich mitansehen mußte, ließ ihn aktiv an sozialen Institutionen mitwirken. Die Gesellschaft zur Beförderung des Guten und Gemeinnützigen wählte ihn im Jahre 1855 zu ihrem Präsidenten. Mit einer Gruppe gleichgesinnter Ärzte war Meyer aktiv an der Gründung der Basler Medizinischen Gesellschaft im Jahre 1860 und an der Gründung der Allgemeinen Krankenpflege (A K P) im Jahre 1863 beteiligt.

Über Jahre hinaus litt Meyer an einer schleichenden Krankheit, die man teils seiner Übermüdung, teils einer Ansteckung durch Typhus zuschrieb. Er erlebte noch die Aufführung seines Samuel Henzi durch die Zofinger im Jahre 1867. Am 5.Dezember des gleichen Jahres erlag er, wie die Untersuchung nach dem Tode ergab, der gleichen Krankheit, der seine Dissertation gegolten hatte: einem Leberkrebs.

\section{Der Arzt und Spitalmeister}

Die beiden Tätigkeiten gehören bei Meyer zweifellos zusammen, obwohl das Amt des Spitalmeisters keine ärztliche Vorbildung verlangte und die ärztliche Nebenbeschäftigung eindeutig ausschloß. Hingegen müssen wir die Berufsfolge, wenn vielleicht auch als zufällige, so doch als logische Ent- 
wicklung in Meyers Leben ansehen. Aus einem Allgemeinpraktiker wurde ein Sozialmediziner, eine für die damalige Zeit höchst ungewöhnliche Berufsgattung und Spezialität.

Ihren Niederschlag findet Meyers einige Jahre dauernde praktische ärztliche Tätigkeit in den «vertrauten Briefen eines Arztes». Aus ihnen spricht Erfahrung in der Heilkunde wie auch Menschenkenntnis. In ihrem Anklang an Paracelsus und in ihrem Wissen, daß nicht nur die Krankheit, sondern der kranke Mensch zu behandeln sei, erlangen sie Zeitlosigkeit. Es handelt sich um insgesamt vier Briefe. Im ersten spricht Meyer vom Tode und der Fragwürdigkeit der ärztlichen Kunst. Der zweite, unbedeutendste, beschreibt die gesellschaftliche Stellung des Arztes. Im dritten wird das Wechselspiel von Leib und Seele beim Kranken beleuchtet. Im vierten schließlich zieht der Autor gegen die Kurpfuscherei los.Aus dem dritten Brief sei teilweise zitiert:

«Ich versichere Dich, oft, ja sehr oft, auch für die Seelen der Kranken sorgen zu müssen, denn viele dieser liegen an den gefährlichsten Krankheiten des inneren Menschen schwer darnieder, und was sie dem Pfarrer nur mit Überwindung und ganz zuletzt offenbaren, wenn der äußerste Rettungshalm abgerissen, das erfährt der Arzt oft ganz leicht, so ganz gelegentlich, weil die Menschen dem, welchem sie vertrauensvoll ihres Leibes Geheimnisse offenbaren, ohne große Mühe auch die der Seele kundgeben. Ich hüte mich immer wohl, die Krankheit der Seele ausdrücklich hervorzuheben, verweile beim lieben theuren Fleische, während ich gleichwohl jenes tiefere Leiden im Auge behalte. Auf dieses aber direkt einzugehen, unaufgefordert, ungefragt, das liegt meiner Natur ferne, und es ist auch nicht meines Amtes. Während ich scheinbar nur für den Leib besorgt bin, lasse ich gleichwohl für die Seele manch gutes Rezeptlein so ganz im Doctorstyl nebenbei mit abfließen. Oft, wenn ich an meiner Gottheit fast verzweifeln wollte und ausrief: es ist all unser Wissen eitel, elendes Stückwerk, Trug und Schein! gab ein solcher Blick in ein so nebenbei gerettetes, getröstetes, aufgerichtetes oder gekräftigtes Gemütsleben mir alle Lust und Kraft wieder. In der Tätigkeit meines ganzen Menschen auf den ganzen Menschen erkannte ich das Göttliche, Segenvolle meines Berufes und hatte Augenblicke wahrer Erhebung.»

Und doch veranlaßten die Zweifel den Arzt, sich nach einer anderen Betätigung umzusehen! Aus allen Briefen spürt man des Autors Ansicht, daß man dem «Publikum» den Willen lassen muß, es aber unbemerkt lenken soll. 
Um einen Eindruck zu bekommen, welche Arbeitslast das Amt des Spitalmeisters oder Spitaldirectors als ausführendes Organ des Pflegamtes mit sich brachte, sei kurz auf die zu Meyers Zeit wirksame Verordnung aus dem Jahre 1825 eingegangen. Der Director soll es sich danach zur Pflicht machen, seine ganze Zeit und Aufmerksamkeit den Geschäften des Spitals zu widmen. Dafür stand ihm freie Wohnung, freie Kost nebst fünf Fässern Wein jährlich und ein Jahresgehalt von 2800 Franken zur Verfügung. Neben der allgemeinen Aufsichtsfunktion galt es, sich mit der Rechnungsführung, mit der Beschaffung von Wein, Lebensmitteln und Holz abzugeben. Es oblag dem Spitalmeister, Erneuerungs- und Renovationsvorschläge einzureichen, Geburten und Todesfälle anzuzeigen, die Kranken aufzunehmen und über jedes Krankenzimmer Buch zu führen. Er war Vertreter des Verwalters und hatte der Spitalverwaltung die Rechnungen vorzulegen. Schließlich hatte er auch für das sittliche und gottesfürchtige Betragen der Spitalinsassen zu sorgen. Bei der Wahl, die für sechs Jahre erfolgte und nur Basler Bürger berücksichtigte, mußte der Neugewählte einen Eid ablegen und eine Kaution von 6000 Franken hinterlegen.

Das Pflichtenheft war umfassend. Manches kleine und große Problem war zu lösen. In seinen Jahresberichten berichtet Meyer unter anderem, wie er die Angestellten zurückhalten mußte, aus dem Spitalgut zu viel herauszupressen. Im gleichen Atemzug warnte er das Armeninstitut vor zu vielen Schmarotzern. Zahlreichen Reklamationen von allen Seiten war nachzugehen. So schienen die Krankenwärter beispielsweise ohne Erlaubnis in der Stadt spazieren zu gehen. Und schließlich - wie könnte es auch anders sein! - beschäftigten ihn schon vor über hundert Jahren Baupläne und Fragen der Ausbalancierung des schon damals defizitären Budgets. Der Verwaltungsbericht vom Jahr 1861 nennt Einnahmen in der Höhe von Fr. 245440.80 und Ausgaben von Fr. 262 771.72, also Mehrausgaben von Fr.17330.92. Wie einfach und fast märchenhaft mutet es heute an, wenn Meyer vorschlug, zur Deckung dieses Defizites sei das tägliche Kostgeld von 75 Rappen auf 80 Rappen zu erhöhen!

Wenn auch Meyer seit der Übernahme des Spitalmeisteramtes keine eigentliche ärztliche Tätigkeit ausübte, so mußte er sich doch mit zahlreichen sozialmedizinischen Fragen auseinandersetzen. Von 1851 bis 1865 tobten in Basel zahlreiche Typhus- und Choleraepidemien. Die damals einzig wirksame Behandlung dieser Seuchen bestand in der Isolierung der Kranken. Dazu brauchte es genügend Spitalbetten. Der zusätzliche Raum wurde in Notspitälern geschaffen. Darüber mußte man aber die Kranken 
überhaupt erst erfassen. Das war weniger einfach, als heute scheint. Gar manche wagten aus Furcht vor den finanziellen Folgen die Krankheit nicht einzugestehen und blieben unerkannte Streuherde. Um diesem Übel abzuhelfen, bemühte sich eine Gruppe von Mitgliedern der im Jahre 1860 gegründeten Medizinischen Gesellschaft intensiv um eine gangbare Lösung. Sie entstand im Jahre 1863 in Form der Allgemeinen Krankenpflege. Es ist verständlich, daß auch der Spitalmeister sich kräftig für die Schaffung dieser für die damaligen Zeiten wegweisenden Krankenkasse einsetzte. Sicherte sie doch gegen bescheidenen Beitrag nicht nur unentgeltlich Arzthilfe und Versorgung mit Medikamenten zu, sondern übernahm auch entstehende Spitalkosten.

Die schönste «ärztliche» Tätigkeit entwickelte Meyer aber in seinen zahlreichen Schriften, auf die im weiteren eingegangen sei.

\section{Der Volksschriftsteller}

Im Todesjahr Meyers erschien seine theoretische Abhandlung Volksschrift und Volksliteratur. Dieser Schrift liegt die interessante These zu Grunde, daß die Dichtung ursprünglich an alle gerichtet und der gesamten Bevölkerung gleich gut verständlich war (Götter- und Heldenlieder). Erst später habe sich zunehmend eine Kluft geöffnet zwischen Gebildeten und Ungebildeten. Diese Trennung zwischen Gelehrten und dem Volke schlechthin habe im 18. Jahrhundert ihren Höhepunkt erreicht. Durch die französische Revolution hätten sich diese Grenzen verwischt. Als Volk sei heute (1860) «jene Classe der Gesellschaft, die mäßig geschult, sich einer mittleren Bildung erfreut» zu verstehen. Für diese Gruppe soll die Volksliteratur geschrieben sein. Diese soll belehrend und unterhaltend zugleich sein. Das Wesentliche sei vom Unwesentlichen zu unterscheiden, ohne daß der Dichter in tendenziöse Verfälschung oder plumpe Schmeichelei verfalle. Das Höchste sei nicht zu hoch und das Edelste nicht zu vornehm für die Volksschrift, denn wohl ist das Volk weniger gescheit als die Gelehrten, hat aber eine gleiche Seele und damit gleiche Empfindungsmöglichkeiten. Der Volksdichter soll es weiterhin verstehen, seine Mitergriffenheit zu zeigen und möglichst oft seinen Humor durchblicken zu lassen. Zwei Stellen aus diesem aufschlußreichen Werk über die Volksdichtung im letzten Jahrhundert seien zitiert:

«dem idelaen Streben aber muß ein ebenso ideales Auffassen des Volkes selbst vorangehen. Bei allem Anschließen an das Volk und gründlichen 


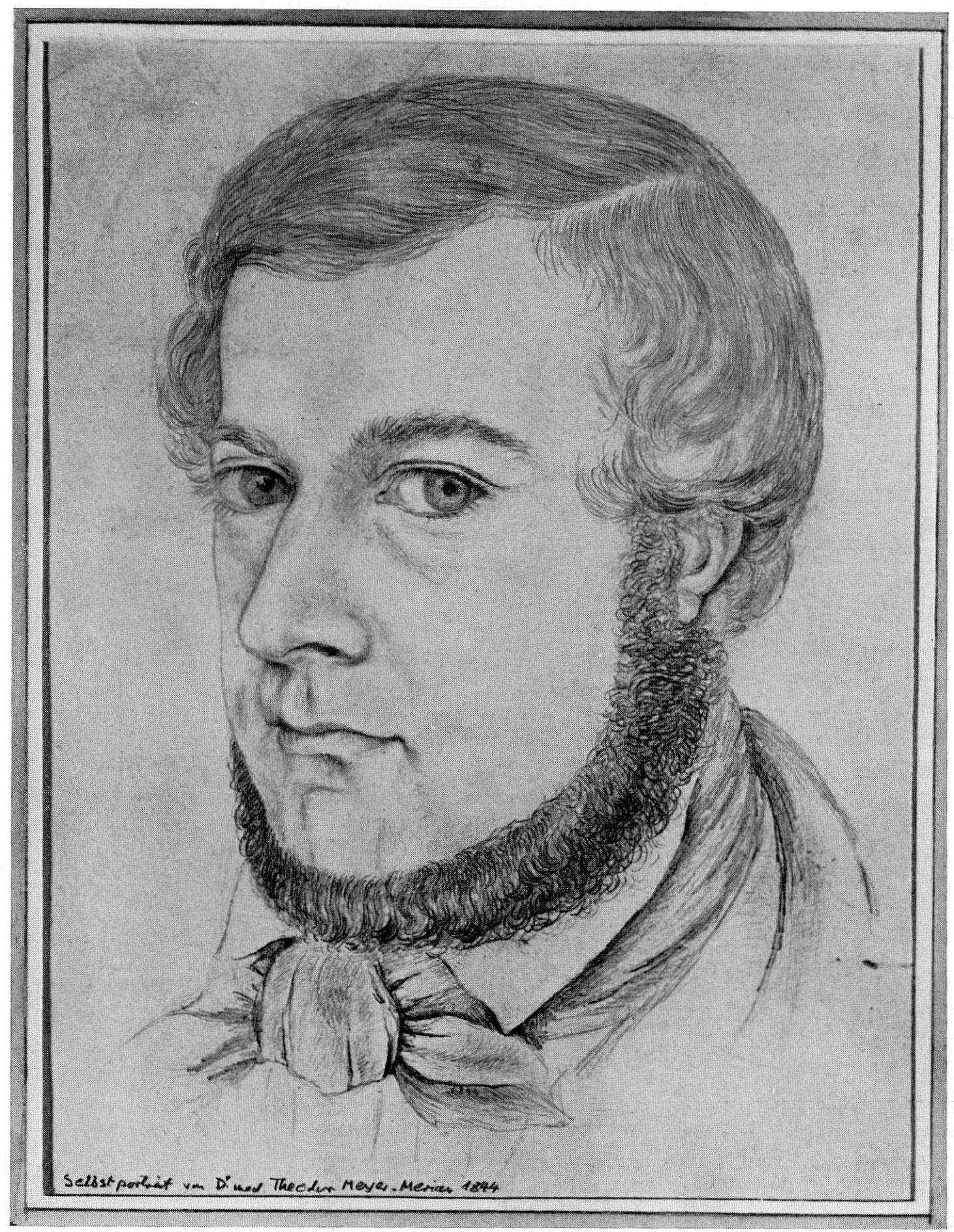

Theodor Meyer-Merian (1818-1867)

Selbstporträt, Bleistift $22 \times 16,5 \mathrm{~cm}$, im Besitze von Prof. Dr. Peter Meyer 


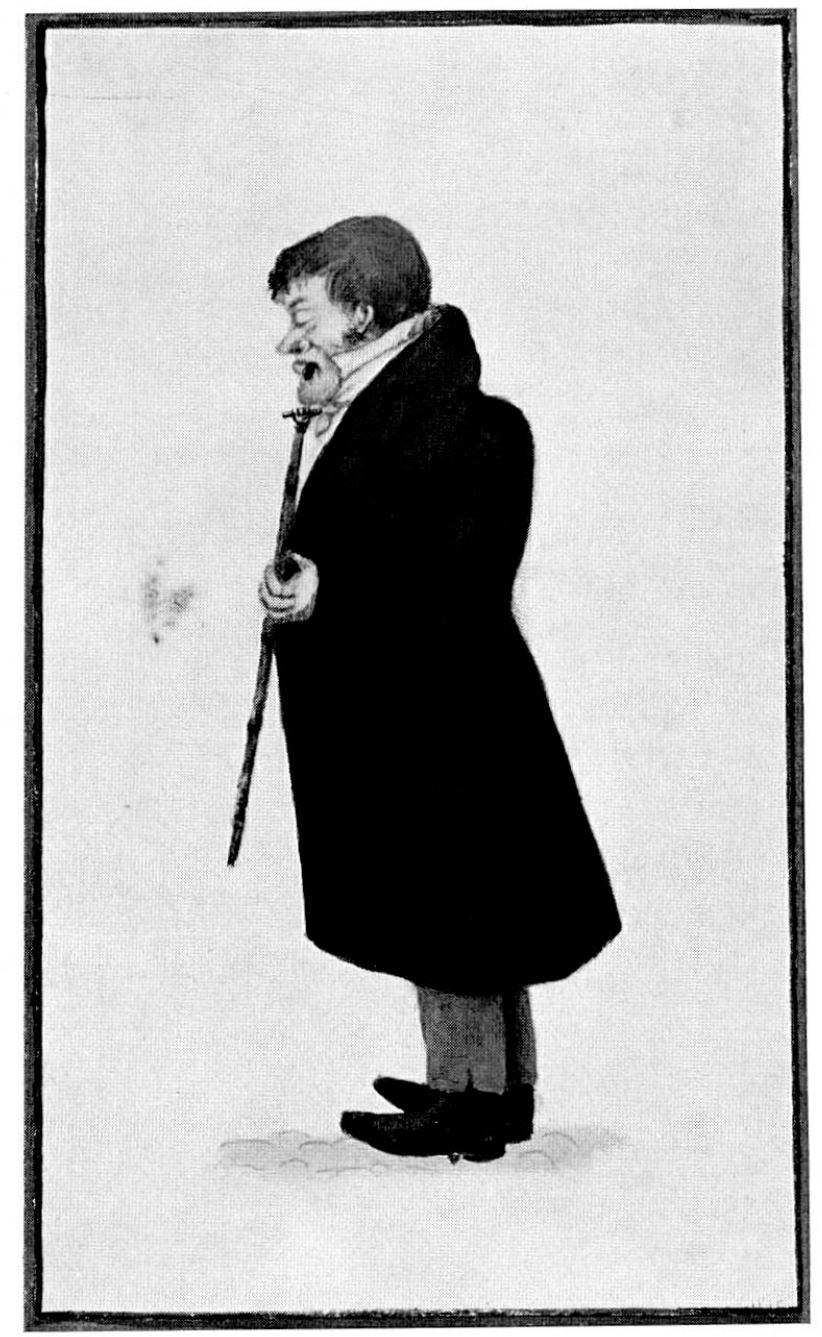

\section{Karikatur von}

Theodor Meyer-Merian:

Christian Friedrich Schönbein

(1799-1868), Professor der Chemie in Basel

\section{Karikatur von}

Theodor Meyer-Merian:

Karl Friedrich Meißner

(1800-1874), Professor der Medizin und der Botanik in Basel

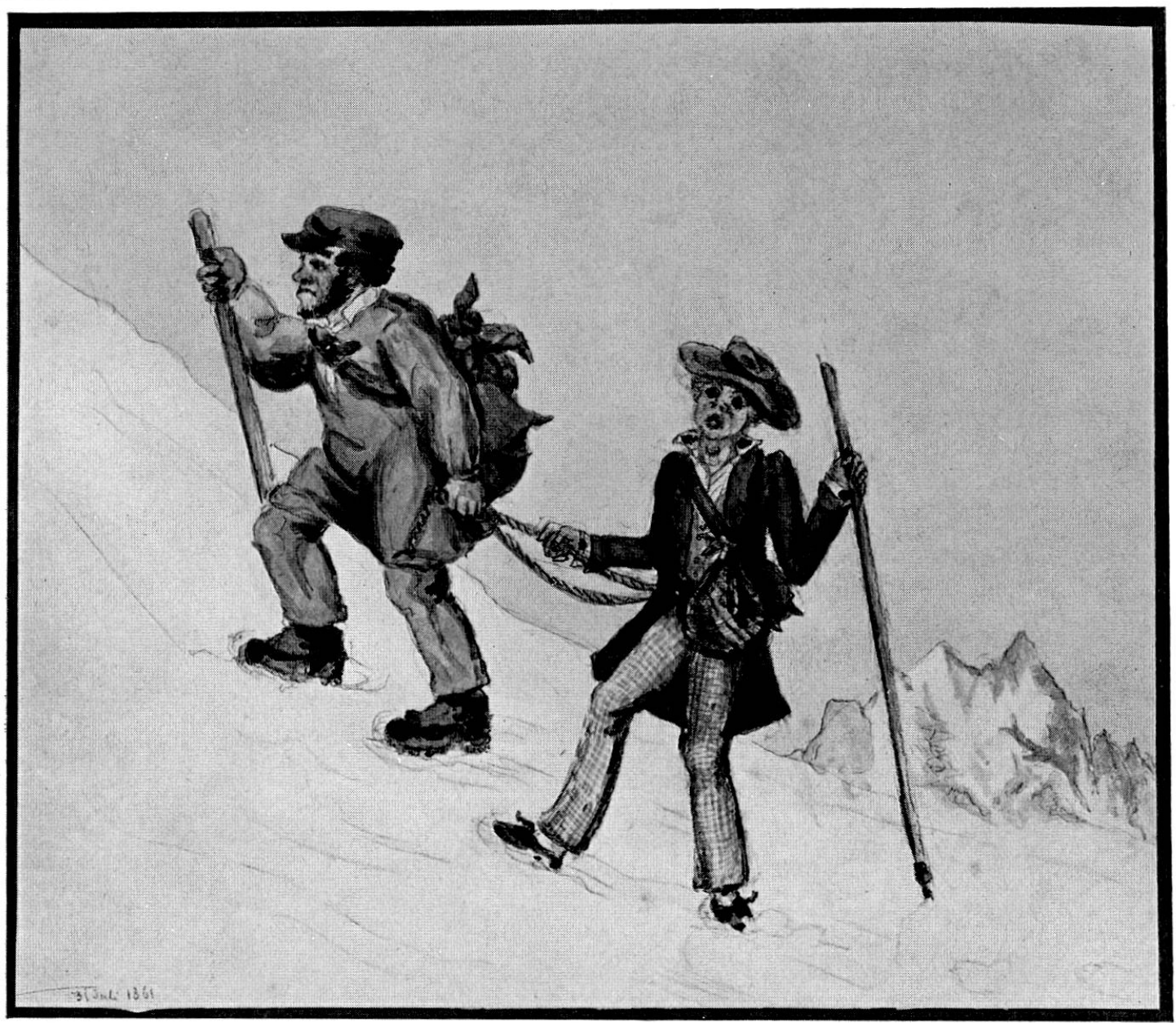


Verstehen desselben, ist auch bei ihm die Voraussetzung eines Idealen in Gesinnung und Tat unerläßlich, mit andern Worten, der Glaube an das Volk und an sein tiefes, ob oft verschleiertes Gefühl für das Höchste, das Ewige.» - Und: «Als den willkommensten Begleiter aber, selbst des tiefen Ernstes, wird das Volk stets den Humor begrüssen, den lächelnden Mund, das treuherzige, wohl gar schalkhafte Auge, welches auch durch den strengen Klang stets das innere Wohlwollen, die Milde, Menschlichkeit des Herzens durchscheinen läßt. Durch das tiefste Volksgemüth zieht sich die Saite einer weisen Ironie gegen die Schwächen alles dessen, was in Erscheinung tritt, mit ein Beweis der im Volke wurzelnden Idealität.»

Aus der umfassenden theoretischen Abhandlung, dem eigentlichen Testament Meyers, läßt sich gut verstehen, daß seine Stärke in der Kleinkunst und -malerei der Kalendergeschichten lag. Sein innerer Drang zur Erziehung des Volkes, welche ihm als Arzt offenbar nicht immer gelungen war, findet hier ihren Niederschlag.

Allerdings hat sich Meyer, wohl unter dem Einfluß des Literaturkränzchens, in allen Dichtungsgattungen versucht: Im Gedicht, Drama, Roman, Aufsatz und Brief. Das Drama (Adalbert Meyer, Winkelried, Alte und Neue Liebe, Samuel Henzi) handelt zumeist von vaterländischen und politischen Ideen. Die Beherrschung der Form ist Meyer hier nie ganz gelungen. Die zum Teil im Dialekt abgefaßten Gedichte (Lieder eines Schweizers [1844], Wintermayeli [1857], Us dr Heimet [1860]) sind wohl hübsch zu lesen, kommen aber in keiner Weise an seine Prosa heran. In ihr hat sich Meyers Begabung erst richtig entfaltet.

Seine Romane sind fast ausschließlich, wie auch die Titel erkennen lassen, sozialen Problemen der Arbeiter- und Handwerkerschichten gewidmet und sind dementsprechend voll erzieherischer Absichten. Um ein paar Beispiele zu nennen: In Der verlorene Sohn, eine Handwerkergeschichte für jedermann (1853) schildert der Dichter das Leben eines haltlosen Handwerksburschen, welcher auszieht, den Gefahren der Großstadt, wie Geltungssucht, Spiel, Prahlerei und Kommunismus (!), begegnet und erliegt. Krank an Leib und Seele kommt er zurück und wird vom treuen Mareili, seiner Jugendfreundin, wieder aufgerichtet. Mareili oder das Bettelmädchen auf dem Letthofe (1860) ist das Kind eines Alkoholikers, dessen Milieu trefflich und abschreckend geschildert wird. Dienen und Verdienen, eine Dienstbotengeschichte (1865) schließlich legt dar, daß der Erfolg mit kleinen Ersparnissen beginnt und hart gegen alle Versuchungen, wie Leichtsinn, Verschwendung und Genußsucht, verteidigt werden muß. 
Vor allem in den späteren Jahren kommt bei Meyer immer mehr das Beschauliche und Idyllische zum Durchbruch. Daß er dabei immer auf dem Boden der Realität bleibt, zeigen uns seine zahlreichen historischen Einleitungen und Abhandlungen, welche den Bienenfleiß des Autors im Zusammentragen von Fakten recht gut erkennen lassen. An rein geschichtlichen Untersuchungen besitzen wir von Meyer eine Geschichte der G G G (1852) und eine Geschichte der Armenherberge Basel (1853). Spannend zu lesen sind die zahlreichen handschriftlichen Notizen, welche Meyer über die Spitalgeschichte zusammengetragen hat und welche noch heute im Staatsarchiv zugänglich sind.

Historischer Sinn, Konservativismus und ein Zug zum Idealen haben Meyer immer mit einer gewissen Wehmut an die Epoche seiner Jugendzeit zurückdenken lassen. Das «entsetzlich sich häutende» Basel erregt in ihm Unbehagen; durch Korrigieren ganzer Straßenzüge schmälert man ihm seine Lieblingsspaziergänge, die erscheinende Eisenbahn vergällt ihm den beschaulichen Nachmittag vor den Toren der Stadt. Gewiß war jene Zeit hektisch und brachte Umwälzungen: Die Tore fielen zum großen Teil und die Mauern, die Stadtgräben wurden aufgefüllt, neue Gebäude entstanden «als hätte die Regierung Tollkraut gefressen». Eine gewisse Scheu vor dem Neuen, vielleicht auch eine gewisse Angst vor dem Ungewissen läßt Meyer immer mehr zurückblicken. In Entschwundene Zeiten (1869) und in Die Nachbarn (1864) hinterläßt er uns herrliche Sittenbilder aus der Biedermeierzeit.

Entschwundene Zeiten schildert uns den Wochenablauf. Da wird der Montag als der griesgrämige beschrieben, mit seiner Neigung, die Leute von der Arbeit fernzuhalten. Dienstag ist der eifrige mit einem besonders gemütlichen Feierabend.Am Mittwoch ist nicht viel los, er gilt als «gar kein Tag». Donnerstag ist der beschauliche. Jedermann zieht am Nachmittag in sein Gärtchen, die weniger Bemittelten in den Stadtgraben, die Reicheren vor die Tore der Stadt. Hier wird gepflanzt, geraucht, getrunken, geplaudert und geträumt. Hier «spann sich ein wesentliches Stück bürgerlichen Daseins ab, halb in ländlicher Arbeit, halb in behaglicher Ruhe, beides zur Erholung und jedenfalls ganz zur Zufriedenheit». Freitag war Markttag, ein Geschehen, welches sich im Zentrum der Stadt abspielte mit einem Stelldichein der Gemüsefrauen, der Käuferinnen, ja überhaupt des ganzen Volkes. Nicht nur Gemüse, auch Nachrichten wurden hier ausgetauscht. Samstag war Putztag. Der «ruhig dahinschreitende Bürger ist in den Straßen seines Lebens nicht sicher vor lauter Klopfen, Bürsten, Kehren, 
Fegen, Wischen, Schütteln». Der Sonntag schließlich ist würdevoll. Sonntagsstaat, Kirchgang und Sonntagsbraten bilden ein unteilbares Ganzes. Der sparsame Bürger entledigt sich schon nach der Kirche seiner schönen Kleider und zieht Hauskleider an. Für den Hausherrn bestehen diese aus Mütze und Hausrock, für die Hausfrau aus Finken und farbiger Hausschürze. Zum Ausgehen wird diese Schürze mit dem schwarzen Fürtuch vertauscht; auf den Kopf kommt die Haartour mit den vier Korkzieherlöckchen, an den Arm die Kommissionentasche («die gewaltige Mappe mit den Tragschnüren») und der zweiplätzige Regenschirm.

Lassen wir Meyer noch einen Blick in die Wohnung werfen ${ }^{3}$ : « Wo noch kein blankgeputzter Messingknopf auf eine Hausglocke deutet, hat die schwere Haustüre noch ihren massiven Türklopfer aus Messing, den man, Einlaß begehrend, stark auf den Nagel fallen läßt. Über die ausgetretene Stufe gelangt man in den kühlen, dunkeln Hausgang und auf der steilen, gewundenen Treppe mit einem Treppenseil statt eines Geländers in den oberen Stock. Der Boden ist stets wie frisch gescheuert, alle Geräte blankgeputzt, überall steht schwerer, alter Hausrat, im Gang ein alter schwarzbrauner Kasten mit hellen Intarsien, der in seiner Tiefe wahre Schätze birgt, in der Stube die geschweifte Nußbaumkommode; am Fenster sind schneeweiße Vorhänge und steht der lackierte Käfig mit dem Kanarienvogel; hier, am Arbeitstischlein mit dem alten, grünen Nähkissen, und draußen, in der Küche, walten die Frauen.» Etwa ist von einer getäfelten Hinterstube die Rede oder von einem grün gemalten Schlafstüblein mit Kupferstichen in schmalen, schwarzen Rähmlein, einem kleinen Spiegel und der Wälderuhr an der Wand. «Der lange Docht der Unschlittkerze im alten Lichtstock ist immer sorgfältig geschneuzt; in Schlafräumen ist sie die einzige Beleuchtung, wozu der Vorrat von vorsorglichen Hausfrauen sorgfältig gelagert wird, während in der Wohnstube über dem Tisch sein Licht ein bescheidenes Lämpchen ausgießt, durch dessen Schirm beim Nachtessen der Dampf der Suppenschüssel zieht. Überall herrscht peinliche Ordnung.»

War das Leben im allgemeinen auch einfach, so boten sich doch immer wieder Gelegenheiten, an welchen ausgelassene Fröhlichkeit mit oft recht derben Spässen hohe Wellen schlagen konnte. In seiner Kalendergeschichte «Das Zunftessen» hat auch hierfür Meyer ein literarisches Denkmal gesetzt.

3 Aus Entschwundene Zeiten. 
In den Kalendergeschichten sind alle Eigenschaften des Dichters zu einer bedeutenden Einheit verbunden: Das historische Denken und die erzieherische Absicht, das beschaulich Idyllische und das beißend Ironische. Die Geschichten drehen sich immer um alltägliche und doch zeitlose Probleme: Die Heirat, die Ehe, das Geld, die Macht, die Sucht usw. Werden im «Zunftessen» die Machtsphären von Mann und Frau festgelegt, so wird in «Herr J.G. Besewind, eine Geschichte aus dem Curleben » ein hypochondrischer Junggeselle, welcher fälschlicherweise in ein Weiberbädlein gerät, auf allopathische Weise geheilt, indem er sich in die Waise einer.ehemaligen Jugendfreundin verliebt. «Vom Kaffee», «Vom Tabak» und «Von Wunderdoktoren» bilden drei genußreiche Aufsätze über drei Artikel, «welche bekanntlich beim Volke in hohem Ansehen stehen». Es würde zu weit führen, hier im einzelnen darauf einzugehen, doch soll der Dichter und Arzt noch einmal zu Worte kommen in einer Geschichte aus dem Schweizerischen Hausboten «Vom Doktern und einer Aderlaßkur». Meyer nimmt hier einen Kollegen aufs Korn, welcher offensichtlich gerne und reichlich zur Ader ließ und ebenso gerne Rezepte verschrieb: «Er hatte manchmal gar eine eigne Art die Kranken, eingebildete und wirkliche, zu kurieren, wie denn überhaupt niemand weniger als er von seinen Mixturen und Pülverchen und Pillen eingenommen war, und er offen mehr als ein Mal sagte: Zwei Drittel von all dem Apothekerzeug könnte man herzhaft auf die Gasse schütten, im Rest fände sich doch noch genug Überflüssiges. Wären nur die Leute nicht so unvernünftig, er würde selten zum Rezepte schreiben kommen und die Apotheker hätten schlechte Tage! - Und der Doktor, der dies sagte, war doch bekannt, ja verschrien dafür bei vielen, daß er wenig verschreibe. Ins Bett liegen und Fasten, das war sein Gewöhnliches.Aber dazu braucht man ja keinen Doktor, man will etwas einnehmen und es ist noch gut, wenn man sich mit einem unschuldigen Tränklein abspeisen läßt. Viele aber begehrten 'Blutreinigungskuren', bei denen man die halbe Seele mit auspurgiert, gleichviel, welchen Weg, und sagte da der Doktor: Nein, so liefen die Leute halt zu einem anderen, der den Brechweinstein mit Kochlöffeln in die Tränklein rühren ließ. Sprach man nun dem Dokter von Solchen, dann sagte er wohl in seiner Art: Freilich, wenn's einen so umstülpt, weiß man, daß inwendig aufgeräumt ist und wieder Platz da und man kann von neuem die alte Art anfangen. Es hält zwar nicht mehr so lange, erst von einem Frühjahr zum andern, dann nur bis zum Herbst, endlich ist's einem alle Monate schlecht, nichts geht von selber, hinten und vorn muß der Dokter, der Apotheker oder sonst ein Geheimmittel stupfen und das Ende 
vom Lied ist ein verhunzter Magen, der nichts mehr erträgt, sondern gleich kapituliert, wenn was beschwerlich fällt. Da heißt's dann, wie nötig und hohe Zeit es gewesen, was für verbranntes Zeug in ihm gesteckt! - Jawohl, verbranntes Zeug, aber im Kopf, nicht im Bauch! Wenn man die Eingeweide wie eine Abfallgrube behandelt, die alle Jahre geleert werden muß, wird sie freilich auch zu einer; dann muß man aber nicht sagen, die Natur verlange das! Nein, die Unnatur.»

\section{Schlußbetrachtung}

Theodor Meyer-Merian steht als Dichter Gotthelf und Hebel nahe, ohne sie je zu erreichen; doch darf ihm der Gehalt an vaterländischer Art und Sitte in seinen Schriften hoch angerechnet werden. Wer die Mühe nicht scheut, sie zu lesen, kann aus ihnen Leben, Gesinnung und Gesittung der Heimat aus der ersten Hälfte des letzten Jahrhunderts zusammentragen, wie sie in solcher Fülle und Echtheit kein Geschichtsbuch zu bieten vermag. Meyer gelang es vor allem, den Geist des unteren Bürgerstandes, der Handwerker und Dienstboten, einzufangen. Aber auch als Arzt ist Meyer eine ungewöhnliche Figur; hat er sich doch zu einer Zeit mit sozialmedizinischen Fragen beschäftigt, in der nur wenige den wirklichen Wert solchen Tuns ermessen konnten. Meyer tritt uns als Arzt und Volksdichter als eine kraftvolle, reiche Persönlichkeit entgegen, für die alle biographische Forschung nur einen schwachen, dürren Eindruck gibt, gemessen an dem lebendigen, sprechenden Werk.

Bibliographie der wichtigsten Werke von Th. Meyer-Merian

chronologisch

a) Druckschriften

Untersuchungen über das Carcinom der Leber, Diss. Basel 1843

Aus den Liedern eines Schweizers, Gedichte, Basel 1844

Adelbert Meyer, Drama, Basel 1851

Über menschlichere Behandlung der Tiere, Preisschreiben, Basel 1851

Geschichte der G G G, 1852

Der verlorene Sohn, eine Handwerkergeschichte für jedermann, Berlin 1853

Die Armenherberge in Basel, in Beiträge zur vaterländischen Geschichte, Basel 1853

Kienseppli oder Almosen und Wohlhaben, Basel 1855

Heinrich Helfenberger oder der Besuch in der Heimat, Basel 1856 
Wintermayeli, Gedichte, Basel 1857

Sicherer Wegweiser zu einer gesunden und guten Wohnung, Basel 1859

Johanna oder Himmel und Erde aus dem Leben eines Weibes, Basel 1858

Us der Heimet, Gedichte, Basel 1860

Mareili oder das Bettelmädchen aus dem Letthofe, Leipzig 1860

Arnold von Winkelried, Drama in fünf Akten, Winterthur 1861

Die Nachbarn, Basel 1864

Dienen und Verdienen, eine Dienstbotengeschichte, Leipzig 1865

Volksschrift und Volksliteratur, Basel 1867

Entschwundene Zeiten, Basel 1869

Heitere Basler Geschichten, Basel 1912

b) Zeitschriften

Der Schweizer Hausbote, Jahrgang 1-4, Basel 1853-1856

Illustrierte Neue Zeitschrift der Schweiz, St. Gallen 1849-1852

(darin die «Vertrauten Briefe eines Arztes»)

Kalender des Basler Hinkenden Boten, Basel 1860-1865

c) Archivakten

Staatsarchiv Basel, Signatur Spital A13 mit handschriftlichen Notizen über die Spitalgeschichte

\section{Literatur über Th. Meyer-Merian}

Burckhardt-Werthemann D., Theodor Meyer-Merian und das Basler Spottbild der Biedermeierzeit, Basler Jahrbuch 1948

Hodel C., Die Anfänge der Krankenversicherung in Basel während des 19. Jahrhunderts und ihre geschichtlichen Voraussetzungen, in Basler Veröffentlichungen zur Geschichte der Medizin und Biologie, Basel 1965

Jenny Ernst, Th. Meyer-Merian. Ein Basler Literatur- und Kulturbild aus dem 19. Jahrhundert, Neujahrsblatt 1919, Basel 1919

Oeri Johann JaKob, Theodor Meyer-Merian, Basel 1870

Oser Friedrich, Theodor Meyer-Merian, Basel 1868 